\title{
Putting Residential Flexibility Management into Action with Pilot Sites in Europe: From Mas2tering to DRIvE Projects ${ }^{+}$
}

\author{
Marie-France Robbe 1,*, Meritxell Vinyals ${ }^{1}$, Stefan Lodeweyckx ${ }^{2}$, Juan Manuel Espeche ${ }^{3}$, \\ Paul-Emmanuel Brun ${ }^{4}$, Sergio Valentino Costa ${ }^{5}$, Monjur Mourshed ${ }^{6}$, Aleksandar Kavgić ${ }^{7}$ and \\ Tatiana Loureiro ${ }^{3}$ \\ 1 CEA, LIST, Metrology, Instrumentation and Information Department, Gif-sur-Yvette CEDEX, \\ 91191 Paris, France; Meritxell.VINYALS@cea.fr \\ 2 Enervalis, Greenville campus, Centrum zuid 1111, 3530 Houthalien-Helchteren, Belgium; \\ stefan.lodeweyckx@enervalis.com \\ 3 R2M Solution, Calle Cervera 59, 1 D, 28033 Madrid, Spain; juan.espeche@r2msolution.com (J.M.E.); \\ tatiana.loureiro@r2msolution.com (T.L.) \\ 4 Airbus Defence and Space, Cassidian cybersecurity SAS, 1 bd Jean Moulin, ZAC de la clef Saint-Pierre, \\ 78890 Elancourt, France; paul-emmanuel.brun@airbus.com \\ 5 COMSA Corporación, Avenida Roma 25, 08029 Barcelona, Spain; sergio.valentino@comsa.com \\ 6 Cardiff University, The Parade, Cardiff CF24 3AA, UK; MourshedM@cardiff.ac.uk \\ 7 Typhoon Hil, Maksima Gorkov 23, 21000 Novi Sad, Serbia; aleksandar.kavgic@typhoon-hil.com \\ * Correspondence: marie-france.robbe@cea.fr; Tel.: +33-1-69-08-87-49 \\ + Presented at Sustainable Places 2018 (SP 2018), Aix-les Bains, France, 27-29 June 2018.
}

Published: 23 August 2018

\begin{abstract}
The Mas2tering and DRIvE European projects develop a software platform to manage the residential and tertiary energy flexibility in local communities of prosumers. This platform includes forecasting, optimization, cybersecurity and fast-response capabilities modules. Business models and use cases were developed, taking into account the regulatory challenges. The platform will be implemented in five pilot sites across three countries.
\end{abstract}

Keywords: residential flexibility; demand-response management; electricity; smart grids

\section{Introduction}

A smart grid has to be conceived as an electric grid able to deliver electricity using an interactive demand-response process between energy producers, distributors and end-users [1]. Domestic final users will be progressively equipped with technologies (e.g., programmable smart appliances, local generation and storage, ...) that are able to provide flexibility. Flexibility is the capacity of controllable smart technologies to modify consumption day-profiles based on external and internal constraints or incentives [2]. The MAS2TERING and DRIvE European projects developed platforms to manage and optimize the flexibility of residential and tertiary consumers to the advantage of both the consumers and the Distribution System Operators (DSO).

2. Mas2tering Project (Multi-Agent Systems and Secured Coupling of Telecom and Energy Grids for Next Generation Smartgrid Services)

The main goal of Mas2tering (2014-2017, FP7 project) is to develop an automatic system that enables to adapt the residential consumption to the local production. The Mas2tering software platform uses the users' flexibility to perform a three-level optimization of electric grid management. 
The home-level optimization maximizes self-consumption or electricity purchase at cheapest tariffs to reduce consumer's energy bill, whereas respecting the user's preferences. The local community optimization enables to balance the local grid by trading local generation surplus between local community's prosumers at tariffs more interesting than those proposed by the corresponding prosumer's supplier. At the low/medium grid level, the optimization enables to smooth the electric consumption during the day, to avoid peaks requiring to build additional production units little used during the year, and to reduce transformer congestions liable to cause a black-out.

The Mas2tering platform is protected by several security components to ensure data privacy, platform security and robustness against vulnerabilities and potential cyber-attacks. The Mas2tering solution has demonstrated potential self-consumption of $99 \%$ at the community level, global peak reduction up to $18 \%$, reduction of grid losses between $5 \%$ and $8 \%$, and potential for substantial energy and carbon savings across the European Union.

\section{DRIvE Project (Demand Response Integration tEchnologies: Unlocking the Demand Response Potential in the Distribution Grid)}

DRIvE (2017-2020, H2020 project) will deliver a fully-integrated, interoperable and secure Demand-Response Management Platform for Aggregators with advanced forecasting, optimization, cyber security, fast-response capabilities and enhanced user participation components. Multi-Agent Systems will move closer to real time operations and progress toward decentralized management of a larger number of assets providing Demand-Response services to prosumers, grid stakeholders and DSOs in a standard-compliant market-regulated manner.

The project features five pilots across three countries consisting of a stadium, wind farm, 7-floor office, tertiary \& residential buildings within medium-large districts, resulting in over $25 \mathrm{MW}$ of potential flexible capacity. 100 households and 2 tertiary buildings over 1000 persons are engaged in the project. The pilots will be running in real DSO environment with real engagement of grid players.

DRIvE will make available average $20 \%$ of load in residential and tertiary buildings for use in Demand-Response, resulting in up to 30\% cost-saving and maximizing revenue for prosumers. DRIvE should allow a minimum $25 \%$ increase of renewable hosting capacity and up to $30 \%$ of overall reduction of CAPEX (capital expenditure) and OPEX (operational expenditure) costs for DSOs.

\section{Regulatory Frameworks Affecting Demand Response Solutions}

Regulatory challenges [3] are critical to the implementation of business cases, and the legislative circumstances surrounding Demand Response are rapidly evolving. Many of these changes are expected to take place before 2020 through the proposed implementation of the Market Design Initiative within the 2016 "Clean Energy for All Europeans" Package (the Winter Package). The Market Design Initiative's proposed directives and regulations focus on supporting prosumers, aggregators and active consumers by facilitating access to Demand Response markets and promoting more market standardization across EU Member States. The DRIvE solution is perfectly positioned to take full advantage of these changes by giving aggregators the tools necessary to enable Demand Response actions in residential and tertiary buildings.

\section{Business Model Opportunities for Local Flexibility Aggregation}

The Local Flexibility Market is presented as a multi-sided platform where the Local Flexibility Aggregator (LFA) is positioned as the 'match-maker' between energy actors needing flexibility for efficiencies and Prosumers engaged in demand response initiatives. The multi-sided business model provides opportunities for numerous stakeholders to exploit through potential business strategies and collaboration opportunities that evolve with transitional phases of the market's maturity. This led to the Mas2tering project storyline, use-cases and associated business cases (BC):

- BC1: Use of the LFA business model opportunity by a Supplier to increase competitiveness, expand its value-added services, retain or grow its client base and answer the call to empower consumers from either Prosumers themselves, regulation or market pressures 
- $\quad$ BC2: Use of the LFA business model opportunity by an existing Wholesale Market Aggregator (WMA) to provide flexibility management services to prosumers within a Local Energy Community to increase its flexibility portfolio and facilitate the formation of Communities;

- $\quad$ BC3: Partnering between a Supplier and WMA to exploit the LFA business model opportunity in a fully functioning flexibility marketplace where the DSO is a flexibility buyer.

Conceptualizing and developing the LFA business model opportunity as a multi-sided platform, is relatively new. Applied to flexibility management, it affords a broad view of the value brought to multiple actors in an electricity market redesign and how flexibility management can become a viable business at the Low-Voltage grid level.

\section{Streaming Data-Based Forecasting for Demand Response}

Accurate forecasting of energy consumption and generation profiles is critical in smart grid management, especially as the electricity grid is expected to host a larger share of variable renewable energy resources such as solar and wind. According to our experience in forecasting algorithms for low-voltage grid management developed in Mas2tering project, the effectiveness of the forecasting algorithms relies on the methods applied and the timeframe/horizon of forecasting. For short-term forecasting, data-driven machine learning algorithms and detailed based simulations are more appropriate than simplified simulations and regression-based models that are more suitable for longterm predictions of energy profiles or single quantity. Occupant behaviour of energy affects the predicted profiles; therefore, further research needs to be carried out to identify ways to integrate the variable in large-scale forecasting. Prediction in intermediate seasons such as Autumn and Spring are more challenging due to their intermittent nature. Most, if not all forecasting models are static and do not account for drift, changes and adaptations in the use of a facility.

Our approach for tackling the challenges of uncertainties in predicted profiles in recently started DRIvE project is to develop a streaming-data based forecasting solution that is better able to consider changes in energy consumption and generation at different time scales.

\section{Multi-Agent System Optimization for Local Energy Communities}

DRIvE and Mas2tering projects develop a decentralised solution based on multi-agent systems to manage residential and tertiary flexibility [4]. Each stakeholder (prosumers, aggregator, etc.) is modelled by a different autonomous agent taking its own decisions, based on its own internal business model and objectives. The coordination of Demand-Response actions (i.e., trading of flexibility) takes place via decentralised negotiation.

The scalable architecture of agent-based technology makes it particularly suitable for DemandResponse solutions, involving multiple optimisation levels. At building level, the Customer Energy Management System (CEMS) agent optimises the use of flexible assets to minimise the prosumer's energy bill and enables bi-directional communication with the energy network to participate in Demand-Response operations. At the community level, the Aggregator agent manages a local decentralised flexibility market (via bi-directional negotiations with each CEMS agent, member of its portfolio) to maximise the self-consumption of local green energy and the revenue of all prosumers. Finally, to prevent occurrence of local congestion in the distribution, a DSO agent can make incentivebased flexibility requests to local aggregators, that in turn negotiate the provision of flexibility with their local markets to reduce the import/export of communities for the congested time-periods.

Results obtained on real-data simulation of local communities showed how Multi-Agent Systems can effectively integrate distributed generation (self-consumption at community level close to $100 \%$ ) whereas maintaining energy security (peak-load and grid losses reduction) and keeping costs down for prosumers (12-15\% savings in the bill). For congestion management, results showed that - in all cases - the investment of DSO is relatively small in comparison with the indicative capital cost of a transformer upgrade. 


\section{Real-Time Controller Hardware in the Loop (CHIL) as the Key Enabling Technology for Next Generation Fast DR}

In addition to shifting loads, the controllable agents of smart assets (primarily inverters, batteries, electric vehicle chargers, HVAC units) can be capable of providing ancillary services to DSOs and balance responsible parties such as voltage control, frequency restoration or containment reserve, and general power quality support. To provide those Fast Demand-Response capabilities, the embedded software must feature local control loops operating at microsecond timesteps i.e., at the timesteps at which switching elements in these assets (inverters, battery chargers) are operating.

The hardware-in-the-loop (HIL) methodology enables to interface real local energy gateways to high-fidelity real-time models (digital twins) of pilot site assets running with a $1 \mu$ s timestep necessary for the ancillary support services. HIL enables to develop, test and optimize nextgeneration demand-response systems in a hybrid environment, where the control software is real but is interfaced to real-time models of the assets. In the DRIvE project, four digital twins of pilot sites will be developed and interfaced to real Local Energy Gateways.

\section{Authentication, Authorization \& Accounting: from Multi-Agent System to Grid Security}

In order to build Flexible Demand Response (DR), connectivity of a growing number of assets is a key enabler. However, increasing asset connectivity, and building cyber physical systems brings cyber threats into the real world. Recent Cyber-Attack consequences, such as the blackout in Kiev in Christmas 2016, show that cybersecurity is a key function to guarantee the resilience of Cyber Physical System. Cyber-attack could have critical impact in terms of safety, cost and even reputation.

In Mas2tering, the cybersecurity challenge was tackled by building a secure-by-design technical architecture, taking into account the main threats of Cyber Physical System. A 3-step security analysis was used: Identification of relevant threats regarding use cases (based on threats databases and ISO 27002); Risk analysis based on ISO/IEC 27005 methodology; Identification of security requirements to implement. Two main security measures were implemented: An early warning system, able to raise alarms based on outdated or vulnerable software; Security components to secure the MultiAgent System platform.

Within DRIvE project, we are targeting to go a step further in security measures implementation by providing an end-to-end security layer within the Demand-Response system, an anomaly detection framework to detect any abnormal cyber or physical behavior, and an investigation capacity to reduce the reaction time

\section{Conclusions}

The demand-side management of residential and tertiary prosumers, organized in local communities supervised by a local aggregator, is an efficient solution to manage the green transition. Flexibility management relies on the capacity to motivate consumers by valorizing the membership to a green community, valuing their flexibility from social and economic aspects [5], and enabling savings on their electricity bills. The software platform, developed by Mas2tering and Drive projects, includes forecasting, optimization, cybersecurity and fast-response capabilities modules. The platform will be implemented in five pilot sites across three countries, to prove its capacity to reduce peak-demand, introduce more intermittent renewable energies in the grid, provide economic benefits for both the consumers and the utilities and deliver ancillary services such as frequency regulation for DSOs.

Funding: Mas2tering (www.mas2tering.eu) and DRIvE (www.h2020-drive.eu) projects have received funding from the European Union's research and innovation programmes (grants agreements FP7 n 619682 and Horizon H2020 n774431). 


\section{References}

1. Siano, P. Demand response and smart grids-A survey. Renew. Sustain. Energy Rev. 2014, 30, 461-478, doi:10.1016/j.rser.2013.10.022.

2. Smale, R.; van Bliet, B.; Spaargaren, G. When social practices meet smart grids: Flexibility, grid management and domestic consumption in The Netherlands. Energy Res. Soc. Sci. 2017, 34, 132-140, doi:10.1016/j.erss.2017.06.037.

3. Good, N.; Ellis, K.A.; Mancarella, P. Review and classification of barriers and enablers of demand-response in the smart grid. Renew. Sustain. Energy Rev. 2017, 72, 57-72, doi:10.1016/j.rser.2017.01.043.

4. Vinyals, M.; Velay, M.; Sisinni, M. A multi-agent system for energy trading between prosumers. In Proceedings of the 14th International Conference on Distributed Computing and Artificial Intelligence (DCAI), Porto, Portugal, June 2017; Volume 620, pp. 79-86, doi:10.007/978-3-319-62410-5.

5. Petersen, M.; Hansen, L.H.; Molbak, T. Exploring the value of flexibility: A smart grid discussion. IFAC Proc. Vol. 2012, 45, 43-48, doi:10.3182/20120902-4-FR-2032.00010.

(C) 2018 by the authors. Licensee MDPI, Basel, Switzerland. This article is an open access article distributed under the terms and conditions of the Creative Commons Attribution (CC BY) license (http://creativecommons.org/licenses/by/4.0/). 